\title{
SUBORDINATION PRINCIPLE AND DISTORTION THEOREMS ON HOLOMORPHIC MAPPINGS IN THE SPACE $C^{n}$
}

\author{
BY \\ KYONG T. HAHN( $\left.{ }^{1}\right)$
}

\begin{abstract}
Generalizing the notion of subordination principle in the complex plane to the space of several complex variables, we obtain various distortion theorems on holomorphic mappings of one bounded domain into another in terms of geometrical quantities of the domains and the Bergman metric furnished, thus obtaining a generalization of the Koebe-Faber distortion theorem among others.
\end{abstract}

1. Introduction. The principle of subordination has been a useful tool in the theory of functions of one complex variable. The idea is that if the range of one holomorphic function is contained in that of a second and these functions agree at a single point then a sharp comparison of these two functions can be made. Naturally, it produces various distortion theorems for holomorphic functions and their coefficients, e.g., see [14].

The main purpose of this paper is to generalize this principle to the space of several complex variables to obtain various distortion theorems and inequalities for certain classes of holomorphic mappings. There seem to be several approaches to do it. The one that we consider here is to take two holomorphic mappings from one domain into another with the same dimensionality. If the range of one mapping is contained in that of a second and these mappings agree at a single point in the domain then some conclusions on distortion of the Jacobian of such mapping functions can be drawn in terms of geometrical quantities of the domains and the metric furnished with it. It also leads to a generalization of Schwarz's lemma to the space of several complex variables ([9], [13]). This is done in $\$ 3$ with some illustrative examples on the classical Cartan domains. In $\$ 2$ we introduce the generalized notion of pseudo-chordal distance and obtain a distortion theorem for holomorphic mappings of a bounded domain into itself in terms of the distance

Presented to the Society, January 23, 1971; received by the editors October 15, 1970 and, in revised form, March 15, 1971.

AMS 1970 subject classifications. Primary 32H10, 32H15; Secondary 32A30.

Key words and phrases. Subordination principle, biholomorphic mapping, generalized Koebe's constant, generalized pseudo-chordal distance, Carathéodory pseudo-metric, classical Cartan domain, bounded symmetric domain, Bergman kernel, Bergman metric, relative invariant, holomorphic automorphism, bounded schlicht domain, homogeneous domain, Koebe-Faber distortion theorem, generalized Vitali's theorem.

(1) Partially supported by NSF Grant GP-11167.

Copyright (C) 1972, American Mathematical Society 
mentioned above. Generalizing the notion of Koebe's constant to the higher dimensional space we obtain a generalization of the so-called Koebe-Faber distortion theorem in $\S 4$.

2. Generalized pseudo-chordal distance. Let $\Delta$ be the unit disk on the complex plane $C$. The Poincaré-Bergman metric on $\Delta$ is given by

$$
\rho(a, b)=\frac{1}{2} \log \frac{1+\delta(a, b)}{1-\delta(a, b)} \text { for } a, b \in \Delta ;
$$

here

$$
\delta(a, b)=|(a-b) /(1-\bar{a} b)|=\tanh \rho(a, b)
$$

is the pseudo-chordal distance between $a$ and $b$ in $\Delta$ [3, Chapter 3].

Let $D$ be a domain in the space $C^{n}$ of $n$ complex variables $z=\left(z_{1}, z_{2}, \ldots, z_{n}\right)$. The Carathéodory (pseudo-) distance of $D$ is defined by

$$
c_{D}(a, b)=\sup _{f \in \mathscr{F}_{D}} \rho(f(a), f(b)) \text { for } a, b \in D,
$$

where $\mathscr{F}_{D}$ denotes the family of holomorphic mappings of $D$ into $\Delta$ ([2], [4]). In general, the Carathéodory distance gives only a pseudo-metric on $D$. But it becomes a (true) metric if and only if the family $\mathscr{F}_{D}$ separates points of $D$. Therefore, $c_{D}$ is a metric if $D$ is bounded. Among the properties that the Carathéodory distance enjoys, the following are useful here:

$\left(\mathrm{P}_{1}\right)$ Every biholomorphic mapping is an isometry, i.e., if $w=w(z)$ is a biholomorphic mapping of $D$ onto $B$, then $c_{D}(a, b)=c_{B}(w(a), w(b))$ for $a, b \in D$.

$\left(\mathrm{P}_{2}\right)$ Every holomorphic mapping of $D$ into $B$ is distance decreasing, i.e., if $w=w(z)$ is a holomorphic mapping of $D$ into $B$, then $c_{D}(a, b) \geqq c_{B}(w(a), w(b))$ for $a, b \in D$.

If $D$ is a bounded domain, the Bergman metric can also be furnished on $D$. It is given by

$$
d s_{D}^{2}=\sum_{\alpha, \beta=1}^{n} T_{\alpha \bar{\beta}} d z_{\alpha} d \bar{z}_{\beta}
$$

where

$$
T_{\alpha \bar{\beta}}=T_{\alpha \bar{\beta}}(z, \bar{z})=\partial^{2} \log K_{D}(z, \bar{z}) / \partial z_{\alpha} \partial \bar{z}_{\beta},
$$

and $K_{D}(z, \bar{z})$ the Bergman kernel function of $D$ [1]. The Bergman metric is a Kähler metric which satisfies property $\left(P_{1}\right)$ but not necessarily $\left(P_{2}\right)$. See [13] for details. The Bergman metric coincides with the Carathéodory metric on the hyperball.

A real valued domain function $Q_{D}(z, \bar{z})$ is a relative invariant of $D$ if it satisfies $Q_{D}(z, \bar{z})=Q_{B}(w, \bar{w})\left|J_{f}(z)\right|^{2}$ under any biholomorphic mapping $w=f(z)$ of $D$ onto $B$, where $J_{f}(z)$ denotes the Jacobian determinant of the mapping. Well-known 
relative invariants are $K_{D}(z, \bar{z})$ and $T_{D}(z, \bar{z})=\operatorname{det}\left(T_{\alpha \bar{\beta}}\right)$. These relative invariants enable us to study geometry of bounded domains.

On a domain $D \subset C^{n}$ we define a distance function $\delta: D \times D \rightarrow[0,1]$ by

$$
\delta_{D}(a, b)=\tanh c_{D}(a, b) \text { for } a, b \in D .
$$

It is clear that $\delta_{D}$ defines a pseudo-metric and satisfies properties $\left(\mathrm{P}_{1}\right)$ and $\left(\mathrm{P}_{2}\right)$. Furthermore, $\delta_{D}$ is a metric if and only if $c_{D}$ is a metric. Clearly, if $D=\Delta \subset C$ then $\delta_{D}$ is the pseudo-chordal distance. If $D$ is the hyperball, $H=\left\{z \in C^{n}:|z|<1\right\}$, $|z|^{2}=(z, z),(z, \zeta)=\sum_{\alpha=1}^{n} z_{\alpha} \zeta_{\alpha}$, then

$$
\delta_{H}(a, b)=\frac{\left(|a-b|^{2}-\left(|a|^{2}|b|^{2}-|(a, b)|^{2}\right)\right)^{1 / 2}}{|1-(a, b)|} \text { for } a, b \in H \quad \text { [6, p. 528]. }
$$

If $D$ is one of the classical Cartan domains $R_{v}, \nu=$ I, II, III, IV, then

$$
\delta_{v}(0, a)=\sup _{|x|=1}|a x| \quad \text { for } a \in R_{v}
$$

where $x$ is an $n$-vector and $|x|$ the length of $x . \delta_{v}(0, a)$ turns out to be the largest among the positive square roots of the characteristic roots of the hermitian matrix $a a^{*}$ [8], where $a^{*}$ denotes the conjugate transpose of the matrix $a$.

In the following we give a distortion theorem for an interior holomorphic mapping. It contains the classical Schwarz's lemma as a special case when $f(0)=0$.

THEOREM 1. Let $D$ be a bounded domain in $C^{n}$ which contains the coordinate origin 0. If $w=f(z)$ is a holomorphic mapping of $D$ into itself, then for $z \in D$

$$
\delta_{D}(0, f(z)) \leqq\left(\delta_{D}(0, f(0))+\delta_{D}(0, z)\right) /\left(1+\delta_{D}(0, f(0)) \delta_{D}(0, z)\right) .
$$

If $D$ is the classical Cartan domain $R_{v}, \nu=\mathrm{I}, \mathrm{II}, \mathrm{III}, \mathrm{IV}$, then

$$
\|f(z)\|_{v} \leqq\left(\|f(0)\|_{\nu}+\|z\|_{v}\right) /\left(1+\|f(0)\|_{v}\|z\|_{v}\right) \quad \text { for } z \in R_{v},
$$

where $\|z\|_{v}=\delta_{v}(0, z)$ as given in (6). If $D$ is the hyperball $H$, then

$$
|f(z)| \leqq(|f(0)|+|z|) /(1+|f(0)||z|) \quad \text { for } z \in H .
$$

Proof. By the triangle inequality applied to the points $0, f(0)$ and $f(z)$ in $D$, $c_{D}(0, f(z)) \leqq c_{D}(0, f(0))+c_{D}(f(0), f(z))$. By $\left(\mathrm{P}_{2}\right), \quad c_{D}(0, f(z)) \leqq c_{D}(0, f(0))+c_{D}(0, z)$. From the addition formula and monotone increasing property of the function tanh, (9) follows. The inequalities (10) and (11) are special cases of (9).

3. Subordination principle and Schwarz's lemma. Let $f$ and $F$ be two holomorphic mappings of a domain $D \subset C^{n}$ into $C^{n}$. We say that $f$ is subordinate to $F$ on $D$ if $F$ is a biholomorphic mapping of $D$ onto $B$ with $f(D) \subset B$, and there exists a point $t \in D$ such that $f(t)=F(t)$. We denote $f<F$ if $f$ is subordinate to $F$, as in the case of one complex variable. 
LEMMA 1. If $f<F$ on a bounded domain $D$ with $f(t)=F(t)$ then there exists a holomorphic mapping $\zeta=\phi(z)$ of $D$ into $D$ with $\phi(t)=t, t \in D$, such that $f=F \cdot \phi$ and $\delta_{D}(t, z) \geqq \delta_{D}(t, \phi(z))$ for $z \in D$.

Proof. Let $F^{-1}: B \rightarrow D$ be the inverse mapping of $F$. Then $\phi(z)=F^{-1}[f(z)]$ defines a holomorphic mapping of $D$ into itself, and $f=F \cdot \phi$. If $f(t)=F(t)$ for $t \in D$, then $\phi(t)=t$. Since the distance $\delta_{D}$ satisfies $\left(\mathrm{P}_{2}\right), \delta_{D}(t, z) \geqq \delta_{D}(t, \phi(z))$ for $z \in D$.

LEMma 2. Let $w=\phi(z)$ be a holomorphic mapping of a bounded schlicht domain $D$ into itself such that there exists $t \in D$ with $\phi(t)=t$. Then, at $t$,

$$
(\partial \phi / \partial z)(\partial \phi / \partial z)^{*} \leqq I_{n}
$$

and equality holds if and only if the mapping $w=\phi(z)$ is an automorphism of $D$, where $(\partial \phi / \partial z)$ denotes the Jacobian matrix of $w=\phi(z)$ and $I_{n}$ the $n \times n$ identity matrix.

Proof (Sketch). In [13] Look uses a matrix analogue of the Cauchy-Schwarz inequality and the reproducing property of the Bergman kernel to show that

$$
(\partial \phi / \partial z)(\partial \phi / \partial z)^{*} \leqq M^{2}\left(T_{\alpha \bar{\beta}}\right) \text { for } z \in D,
$$

where $M=\sup _{D}|\phi(z)|$. Since $\left(T_{\alpha \bar{\beta}}\right)$ is positive definite, there exists a positive number $a$ such that

$$
\left(T_{\alpha \bar{\beta}}\right)_{t} \leqq a I_{n} .
$$

Thus, $A \equiv(\partial \phi / \partial z)_{t}(\partial \phi / \partial z)_{t}^{*} \leqq a M^{2} I_{n}$. Since $\phi(t)=t$, it holds that $A^{k} \leqq a M^{2} I_{n}$ for every positive integer $k$. Since $A$ is hermitian, there exists a basis for which

$$
A=\left(\begin{array}{llll}
\mu_{1} & & & 0 \\
& \cdot & & \\
& & \cdot & \\
0 & & & \mu_{n}
\end{array}\right)
$$

with each $\mu_{j}>0$. Hence for each $k, \mu_{j}^{k} \leqq a M^{2}$ which implies $\mu_{j} \leqq 1$. So $A \leqq I_{n}$.

From this lemma we have

THEOREM 2. If $f<F$ on a bounded schlicht domain with $f(t)=F(t)$ for $t \in D$, then at $t$

$$
(\partial f / \partial z)(\partial f / \partial z)^{*} \leqq(\partial F / \partial z)(\partial F / \partial z)^{*}
$$

and equality holds if and only if the mapping $w=F^{-1} \cdot f(z)$ is an automorphism of $D$ with $t$ as a fixed point. In particular,

$$
\left|J_{f}(t)\right| \leqq\left|J_{F}(t)\right|,
$$

where $J_{f}(t)$ denotes the Jacobian determinant of $w=f(z)$ at the point $t$. 
Proof. By Lemma 1, there exists a holomorphic mapping $w=\phi(z)$ of $D$ into itself such that $\phi(t)=t$ and $f=F \cdot \phi$. By the chain rule, $(\partial f / \partial z)=(\partial F / \partial \zeta)(\partial \phi / \partial z)$, $\zeta=\phi(z)$, and hence, the theorem follows from Lemma 2 . Inequality (5) follows trivially from (4).

Theorem 3. Let $D$ be a bounded homogeneous schlicht domain in $C^{n}$ and $0 \in D$. If $f$ is a holomorphic mapping of $D$ into itself, then for $z \in D$

$$
\left|J_{f}(z)\right|^{2} \leqq T_{D}(z, \bar{z}) / T_{D}\left(f(z), f(z)^{-}\right)
$$

and equality holds if and only if $f$ is an automorphism of $D$.

Proof. Let $t$ denote the holomorphic automorphism of $D$ which maps the origin 0 into $t \in D$. Then $F=\tau \cdot t^{-1}$ is a holomorphic automorphism of $D$ which maps $t$ into $\tau=f(t)$ and $F(t)=f(t)$. Thus $f<F$ with $f(t)=F(t)$. Since $T_{D}$ is a relative invariant of $D, T_{D}(0,0)\left|J_{t}-1(t)\right|^{2}=T_{D}(t, \bar{t})$ and $T_{D}(\tau, \bar{\tau})\left|J_{\tau}(0)\right|^{2}=T_{D}(0,0)$. The theorem now follows from (5) when we observe $J_{F}(t)=J_{\tau}(z) J_{t}-1(t)$. Since $F$ is an automorphism of $D$, equality in (6) holds if and only if $f$ is an automorphism of $D$, by Theorem 2 .

This gives a different proof of Theorem 1 in [9] when $\Delta=D$.

REMARK 1. Using Theorem 3 and the results in $[9, \S 4]$ we obtain an explicit upper bound for the Jacobian determinant of the interior mapping $w=f(z)$ of the classical Cartan domain $R_{v}, \nu=\mathrm{I}$, II, III, IV, e.g., for $R_{I}$

$$
\left|J_{f}(z)\right| \leqq\left[\operatorname{det}\left(I-w w^{*}\right) / \operatorname{det}\left(I-z z^{*}\right)\right]^{(m+n) / 2}, \quad w=f(z),
$$

and for the hyperball $H$

$$
\left|J_{f}(z)\right| \leqq\left(\left(1-|f(z)|^{2}\right) /\left(1-|z|^{2}\right)\right)^{(n+1) / 2} .
$$

THEOREM 4. Let $D$ be a bounded symmetric domain, starshaped with respect to $0 \in D$. If $f<F$ on $D$ with $f(0)=F(0)$, then for $z \in D$

$$
\left|J_{f}(z)\right| \leqq\left|J_{F}(0)\right|^{P(z)} \text {, }
$$

where $P(z)=\max _{B} P(z, t)$ is the Poisson kernel of $D([11],[12])$ and $B$ the BergmanSilov boundary of $D$. In particular, if $D$ is the classical Cartan domain $R_{v}, \nu=I$, II, III, IV, then for $z \in R_{v}$

$$
\left|J_{f}(z)\right| \leqq\left|J_{F}(0)\right|^{\left.\left(1+\|z\|_{v}\right) /\left(1-\|z\|_{v}\right)\right) p v}
$$

where $p_{v}$ denotes the (complex) dimension of $R_{v}$ and \|\|$_{v}$ the generalized pseudochordal distance on $R_{v}$.

Proof. Since $F$ is a biholomorphic mapping of $D$, the Jacobian $J_{F}(z)$ omits the value 0 and hence $\log J_{F}(z)$ defines a holomorphic function on $D$. Since $D$ is starshaped with respect to $0 \in D, \log J_{F}(r z), 0<r<1$, is holomorphic in $D$. By (4.8.3) of [11]

$$
\log J_{F}(r z)=\int_{B} P(z, b) \log J_{F}(r b) d b,
$$


where $d b$ is the circularly invariant normalized measure on $B$. For each $z \in D$ the Poisson kernel $P(z, b)$ is continuous on $\bar{D}$. So $P(z)=\max P(z, b)$ exists on $B$. Taking the real parts in (11) we obtain

$$
\begin{aligned}
\log \left|J_{F}(r z)\right| & =\int_{B} P(z, b) \log \left|J_{F}(r b)\right| d b \\
& \leqq P(z) \int_{B} \log \left|J_{F}(r b)\right| d b=P(z) \log \left|J_{F}(0)\right| .
\end{aligned}
$$

The last inequality follows from (11) and the fact that $P(0, b)=1[11, \mathrm{p} .98]$.

Letting $r \rightarrow 1$ we have (9) from Theorem 2 and (12). In particular, for the classical Cartan domains $R_{v}$ the bounds for the Poisson kernels are explicitly given by

$$
Q_{v}(z) \leqq P(z, b) \leqq Q_{v}(z)^{-1}, \quad Q_{v}(z)=\prod_{k=1}^{n_{v}}\left(\frac{1-\lambda_{k}}{1+\lambda_{k}}\right)^{N_{v}},
$$

where $n_{\mathrm{I}}=m, n_{\mathrm{II}}=n, n_{\mathrm{III}}=[n / 2], n_{\mathrm{IV}}=2 ; N_{\mathrm{I}}=n, N_{\mathrm{II}}=(n+1) / 2, N_{\mathrm{III}}=n-1$, if $n$ is even, and $=n$ if $n$ is odd, $N_{\mathrm{IV}}=n / 2 ; \lambda_{1}, \lambda_{2}, \ldots, \lambda_{n_{v}}$ are the nonnegative square roots of the characteristic roots of the hermitian matrix $z z^{*}$ for $z \in R_{v}$, and $1>\lambda_{1} \geqq \cdots$ $\geqq \lambda_{n_{v}} \geqq 0$. See [8] for example. Since the function $(1+t) /(1-t)$ is an increasing function of $t$, we have the following inequalities:

$$
P_{v}(z)=\max _{B_{v}} P(z, b) \leqq Q_{v}(z)^{-1} \leqq\left(\frac{1+\lambda_{1}}{1-\lambda_{1}}\right)^{n_{v} N_{v}},
$$

where $B_{v}$ denotes the Bergman-Silov boundary of $R_{v}$. By (2.8) $\lambda_{1}$ is the generalized pseudo-chordal distance from 0 to $z$, and we have (10) from (9), (14) and the fact that $p_{v}=n_{v} N_{v}$ is the dimension of $R_{v}$.

Suppose that $w=f(z)$ is a holomorphic mapping of a bounded symmetric domain $D$ into itself. If $w=F(z)$ is a holomorphic automorphism of $D$ which maps $0 \in D$ into $f(0)$, then $f<F$ with $f(0)=F(0)$. By Theorem $4,\left|J_{f}(z)\right| \leqq\left|J_{F}(0)\right|^{P(z)}$ for $z \in D$. Since the Bergman kernel function $K_{D}(z, \bar{z})$ is a relative invariant [1, Chapter 11] of $D,\left|J_{F}(0)\right|^{2}=K_{D}(0,0) / K_{D}\left(f(0), f(0)^{-}\right)$. It is well known [11, p. 82] that $K_{D}(0,0)$ $=1 / v$, where $v$ denotes the euclidean volume of $D$. Therefore, we obtain the following corollary.

COROLlaRY 1. Let $D$ be a bounded symmetric domain, starshaped with respect to $0 \in D$. If $w=f(z)$ is a holomorphic mapping of $D$ into itself, then for $z \in D$

$$
\left|J_{f}(z)\right| \leqq\left[v K_{D}(a, \bar{a})\right]^{-P(z) / 2}, \quad a=f(0) .
$$

In particular, if $D$ is the classical Cartan domain $R_{v}, \nu=\mathrm{I}$, II, III, IV, then for $z \in R_{v}$, $\nu=$ I, II, III,

$$
\left|J_{f}(z)\right| \leqq\left[\operatorname{det}\left(I-a a^{*}\right)\right]^{e_{\nu}}
$$

and for $R_{\mathrm{IV}}{ }^{\prime}$

$$
\left|J_{f}(z)\right| \leqq\left(1+\left|a a^{\prime}\right|^{2}-2 \bar{a} a^{\prime}\right)^{e_{\mathrm{IV}}}
$$

where $e_{v}=\left(l_{v} / 2\right)\left[\left(1+\|z\|_{v}\right) /\left(1-\|z\|_{v}\right)\right]^{p_{v}}, l_{\mathrm{I}}=m+n, l_{\mathrm{II}}=n+1, l_{\mathrm{III}}=n-1$ and $l_{\mathrm{IV}}=n$. 
For the explicit expressions for the kernel functions of the classical Cartan domains, see [9], [11].

4. Generalization of the Koebe-Faber distortion theorem. Let $D$ be a bounded schlicht domain in the space $C^{n}$ which is starshaped with respect to the origin $0 \in D$, i.e., $z \in D$ implies $r z \in D$ for $r \in[0,1]$.

THEOREM 5. Let $D$ be a bounded homogeneous schlicht domain in $C^{n}$ which is starshaped with respect to the origin $0 \in D$. If $w=f(z)$ is a biholomorphic mapping of $D$ onto $f(D)$, then for $z \in D$

$$
\left|J_{f}(z)\right| \geqq d_{w}^{n}\left[T_{D}(z, \bar{z}) / T_{D}(0,0)\right]^{1 / 2}=d_{w}^{n}\left|J_{z}(0)\right|^{-1},
$$

where $d_{w}$ denotes the largest number such that $w+d_{w} \zeta \subset f(D)$ for all $\zeta \in D$ and $J_{z}$ the Jacobian determinant of an automorphism which maps 0 into z. Furthermore, let $R_{D}$ be the radius of the smallest hyperball containing $D$. Then for $z \in D$

$$
(\partial f / \partial z)^{*}(\partial f / \partial z) \geqq\left(d_{w}^{2} / R_{D}^{2} \lambda_{D}^{2}(0)\right) I_{n},
$$

where $\lambda_{D}(0)$ denotes the positive square root of the largest characteristic root of the metric tensor $\left(T_{\alpha \bar{\beta}}\right)$ at the point 0 .

Proof. Since $w=f(z)$ is a biholomorphic mapping of $D$ onto $f(D)$, there exists an inverse mapping $z=f^{-1}(w)$ from $f(D)$ onto $D$. Let

$$
\eta=h(\zeta)=f^{-1}\left(w+d_{w} \zeta\right) \quad \text { for } \zeta \in D .
$$

Then $h$ is a biholomorphic mapping of $D$ into itself with $h(0)=f^{-1}(w)=z$. Furthermore, $J_{h}(\zeta)=J_{f^{-1}}(\tau) \cdot J_{\tau}(\zeta), \quad \tau=w+d_{w} \zeta$. Since $J_{\tau}(\zeta)=d_{w}^{n}, J_{f^{-1}}(\tau)=1 / J_{f}(\eta)$ and $\eta=h(0)=z$ for $\zeta=0$, we have

$$
J_{h}(0)=d_{w}^{n} / J_{f}(z)
$$

By Theorem $3,\left|J_{h}(\zeta)\right|^{2} \leqq T_{D}(\zeta, \zeta) / T_{D}\left(h(\zeta), h(\zeta)^{-}\right)$for $\zeta \in D$. In particular, for $\zeta=0$,

$$
\left|J_{h}(0)\right|^{2} \leqq T_{D}(0,0) / T_{D}\left(h(0), h(0)^{-}\right)=T_{D}(0,0) / T_{D}(z, \bar{z}) .
$$

Since $D$ is homogeneous and $T_{D}(z, \bar{z})$ is a relative invariant of $D$, we have

$$
T_{D}(z, \bar{z}) / T_{D}(0,0)=K_{D}(z, \bar{z}) / K_{D}(0,0)=\left|J_{z}(0)\right|^{-2} \text {. }
$$

(1) follows from (4), (5) and (6). From (3) we have

$$
(\partial h / \partial \zeta)_{0}=d_{w}(\partial f / \partial z)^{-1}
$$

where $(\partial h / \partial \zeta)_{0}$ means the Jacobian matrix evaluated at $\zeta=0$. By the Fundamental Theorem 1 of Look [13], see also (3.2),

$$
(\partial h / \partial \zeta)_{0}(\partial h / \partial \zeta)_{0}^{*} \leqq R_{D}^{2}\left(T_{\alpha \bar{\beta}}\right)_{0} .
$$

(2) follows from (7), (8) and the inequality: $\left(T_{\alpha \bar{\beta}}\right)_{0} \leqq \lambda_{D}^{2}(0) I_{n}$. 
Applying the inequality (1) to the classical Cartan domains we have

COROLlary 2. Let $w=f(z)$ be a biholomorphic mapping of the classical Cartan domains $R_{v}, \nu=$ I, II, III, IV. Then for $z \in R_{v}, \nu=$ I, II, III,

$$
\left|J_{f}(z)\right| \geqq d_{w}^{p_{v}}\left[\operatorname{det}\left(I-z z^{*}\right)\right]^{-l_{v} / 2},
$$

where $l_{v}$ is given as in (3.14) and $p_{v}$ the dimension of $R_{v}$, and for $z \in R_{\mathrm{IV}}$

$$
\left|J_{f}(z)\right| \geqq d_{w}^{n}\left[1+\left|z z^{\prime}\right|^{2}-2 \bar{z} z^{\prime}\right]^{-n / 2} .
$$

By $\mathscr{S}(t)$ we denote the family of biholomorphic mappings $w=f(z)$ of $D$ into a bounded domain, normalized at $t \in D$ by $f(t)=0$ and $J_{f}(t)=1$.

LEMMA 3. There exists a positive number $\rho$ such that for each $f \in \mathscr{S}(t), D_{\rho} \subset f(D)$, where $D_{\rho}=\{\rho z: z \in D\}$.

Proof. Suppose that the lemma is false. Then for each positive integer $k$ there exists an $f^{(k)} \in \mathscr{S}(t)$ such that the number $\rho_{k}$ defined above is less than $1 / k$. Applying the generalized Vitali theorem [5], we see that there exists a subsequence $f^{(l)}$ which converges to a holomorphic mapping $g: D \rightarrow C^{n}$ uniformly on compact subsets of $D$. Clearly, there is no positive number $\rho$ with the property $D_{\rho} \subset g(D)$. Since uniform convergence of holomorphic functions implies uniform convergence of the corresponding partial derivatives of all orders, the sequence of Jacobian determinants $J_{f}^{(l)}(z)$ converges to $J_{g}(z)$ uniformly on compact subsets of $D$, and hence, $J_{g}(t)=1$. Therefore, there exists a neighborhood of $t$ which can be mapped by $w=g(z)$ onto a neighborhood of the origin 0 biholomorphically, i.e., there exists a positive number $\rho$ such that $D_{\rho} \subset g(D)$. It is impossible.

Lemma 3 secures the existence of the largest positive number $r$ such that $D_{r}$ $\subset \bigcap_{f \in \mathscr{S}(t)} f(D)$. We denote this number by $r_{t}(D) . r_{0}(D)$ is a generalization of the Koebe constant in the complex plane to the higher dimensional space.

Finally, we generalize the Koebe-Faber distortion theorem in the complex plane, see [15, p. 145] for example, to the bounded homogeneous domain in the space $C^{n}$.

THEOREM 6. Let $r_{0}(D)$ be the generalized Koebe constant of the bounded homogeneous schlicht domain $D, 0 \in D$. If $f \in \mathscr{S}(t)$ and if $w=f(z)$, then

$$
\left|J_{f}(z)\right| \leqq \frac{d_{w}}{r_{0}(D)}\left[\frac{T_{D}(z, \bar{z})}{T_{D}(0,0)}\right]^{1 / 2}=\frac{d_{w}}{r_{0}(D)}\left|J_{z}(0)\right|^{-1} \quad \text { for } z \in D .
$$

Proof. If $f$ is a biholomorphic mapping of $D$, then $g(z)=(f(z)-f(t)) / J_{f}(t)$ belongs to the class $\mathscr{S}(t)$. Hence, by the definition of $r_{t}(D), g(D)$ contains $D_{r_{t}}$, and hence, $f(D)$ contains $D_{r_{t}\left|J_{f}(t)\right|}+f(t), r_{t}=r_{t}(D)$. Let $h=f \cdot z \cdot t^{-1}$, where $z$ and $t$ denote the automorphisms of $D$ which map 0 into $z$ and $t$, respectively. Then $h$ is a biholo- 
morphic mapping of $D$. As before, $h(D)$ contains $D_{r_{t}\left|J_{h}(t)\right|}+h(t)=f(z)+D_{r_{t}\left|J_{h}(t)\right|}$. Since $\left|J_{h}(\zeta)\right|^{2}=\left|J_{f}(w)\right|^{2}\left|J_{z}(\eta)\right|^{2}\left|J_{t^{-1}}(\zeta)\right|^{2}, w=z(\eta), \eta=t^{-1}(\zeta)$,

$$
\left|J_{h}(t)\right|=\left|J_{f}(z)\right|\left[T_{D}(t, \bar{t}) / T_{D}(z, \bar{z})\right]^{1 / 2} .
$$

This means that

$$
d_{w} \geqq r_{t}\left|J_{f}(z)\right|\left[T_{D}(t, \bar{t}) / T_{D}(z, \bar{z})\right]^{1 / 2} .
$$

In particular, for $t=0,(11)$ follows.

For the classical Cartan domains, we have the following corollary:

COROLlary 3. Let $r_{v}$ be the generalized Koebe constant for the classical Cartan domains $R_{v}, \nu=\mathrm{I}$, II, III, IV. If $f \in \mathscr{S}(0)$ and if $w=f(z)$, then for $z \in R_{v}, \nu=\mathrm{I}$, II, III,

$$
\left|J_{f}(z)\right| \leqq\left(d_{w} / r_{v}(D)\right)\left[\operatorname{det}\left(I-z z^{*}\right)\right]^{-l_{v} / 2},
$$

and for $z \in R_{\mathrm{IV}}$

$$
\left|J_{f}(z)\right| \leqq\left(d_{w} / r_{\mathrm{IV}}(D)\right)\left[1+\left|z z^{\prime}\right|-2 \bar{z} z^{\prime}\right]^{-n / 2} .
$$

\section{REFERENCES}

1. S. Bergman, The kernel function and conformal mapping, 2nd rev. ed., Math. Surveys, no. 5, Amer. Math. Soc., Providence, R. I., 1970.

2. C. Carathéodory, Uber das Schwarsche Lemma bei analytischen Funktionen von zwei komplexen Veränderlichen, Math. Ann. 97 (1927), 76-98.

3. - Theory of functions of a complex variable. Vol. 2, Chelsea, New York, 1954. MR 16, 346.

4. B. A. Fuks, Special chapters in the theory of analytic functions of several complex variables, Fizmatgiz, Moscow, 1963; English transl., Transl. Math. Monographs, vol. 14, Amer. Math. Soc., Providence, R. I., 1965. MR 30 \#4979; MR 32 \#5915.

5. R. C. Gunning and H. Rossi, Analytic functions of several complex variables, Prentice-Hall, Englewood Cliffs, N. J., 1965. MR 31 \#4927.

6. K. T. Hahn, The non-euclidean Pythagorean theorem with respect to the Bergman metric, Duke Math. J. 33 (1966), 523-534. MR 34 \#6149.

7. - Some properties of relative invariants on bounded domains, Duke Math. J. 34 (1967), 325-332. MR 35 \#5658.

8. - A remark on integral functions of several complex variables, Pacific J. Math. 26 (1968), 509-513. MR 38 \#1284.

9. K. T. Hahn and J. Mitchell, Generalization of Schwarz-Pick lemma to invariant volume in a Kähler manifold, Trans. Amer. Math. Soc. 128 (1967), 221-231. MR 39 \#4444.

10. - $H^{p}$ spaces on bounded symmetric domains, Trans. Amer. Math. Soc. 146 (1969), 521-531. MR 40 \#6247.

11. L. K. Hua, Harmonic analysis of functions of several complex variables in the classical domains, Science Press, Peking, 1958; English transl., Transl. Math. Monographs, vol. 6, Amer. Math. Soc., Providence, R. I., 1963. MR 23 \#A3277; MR 30 \#2162.

12. A. Korányi, The Poisson integral for generalized half-planes and bounded symmetric domains, Ann. of Math. (2) 82 (1965), 332-350. MR 34 \#371. 
13. K. H. Look, Schwarz lemma and analytic invariants, Sci. Sinica 7 (1958), 453-504. MR 21 \#5028.

14. W. W. Rogosinski, On the coefficients of subordinate functions, Proc. London Math. Soc. (2) 48 (1943), 48-82. MR 5, 36.

15. W. A. Veech, A second course in complex analysis, Benjamin, New York, 1967. MR 36 \#3955.

16. V. S. Vladimirov, Methods of the theory of functions of several complex variables, "Nauka", Moscow, 1964; English transl., M.I.T. Press, Cambridge, Mass., 1966. MR 30 \#2163; MR 34 \#1551.

Pennsylvania State University, University Park, Pennsylvania 16802 